
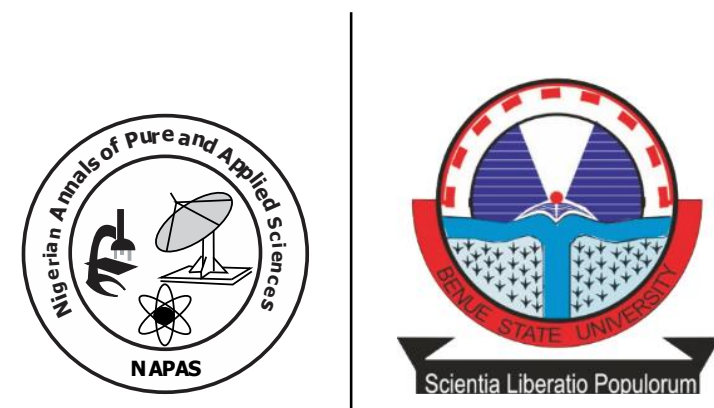

\title{
The Effect of Internal Heat Generation of a Reactive Hydromagnetic (MHD) Fluid Flow through a Vertical Plate
}

\section{Ikpakyegh, L. N. 1, Shio, M. M. r², Okedayo, G.T. ${ }^{3}$,}

1Department of Mathematics/Statistics/Computer Science, University of Agriculture Makurdi, Benue State, Nigeria

E-mail address.lewisnyitor@yahoo.com

${ }^{2}$ department of Mathematics/Computer Sciences, Benue State University Makurdi, Benue State, Nigeria

E-mail address.talk2msuurshior@ymail.com

${ }^{3}$ department of Mathematical Sciences, Ondo State University of Science and

Technology Okitipupa, Ondo State, Nigeria

E-mail address.okedayo@yahoo.com

\begin{abstract}
In this study, we discussed the flow of an electrically conducting fluid with internal heat generation over a vertical plate on a reactive MHD fluid. We obtained the semi analytical solutions of the dimensionless equations governing the fluid flow using the method of weighted residual by the collocation method. The parameters with their effect on velocity and temperature fields are discussed graphically using Mapple 17 computer software. We showed that the internal heat generation, magnetic field intensityand the reactive rate have significant effects on the velocity profiles and temperature distributions.
\end{abstract}

Key words: Magnetohydrodynamics (MHD), vertical plate, reactive fluid, internal heat generation. 


\section{Introduction}

Studies relating to flow of fluids between two parallel plates are on the increase in recent time due to its numerous applications in many industrial and engineering processes like in accelerators, aerodynamic heating, polymer technology, purification of crude oil and magnetohydrodynamics (MHD) generators. The study of heat generation or absorption effects in moving fluids is important in view of several physical problems, such as fluids undergoing exothermic and endothermic chemical reactions (Hassan and Gbadeyan, 2015).

Reactive hydro- magnetic fluid flows are often accompanied with heat transfer in many industrial and engineering applications. For instance, Makinde and Beg (2010) devoted their study to investigate the inherent irreversibility and thermal stability in a reactive electrically conducting fluid flowing steadily through a channel with isothermal walls under the influence of a transversely imposed magnetic field. Recently, Hassan and Gbadeyan (2015) investigated the entropy generation analysis of a reactive hydro-magnetic fluid flow through a channel with isothermal wall temperature under different chemical kinetics without taking into account the effects of internal heat generation within the flow system.

Lot of research works concerning the fluid flow between two vertical plates has been obtained under different physical and chemical conditions. Possible heat generation effects may alter the temperature distribution as well as the particle deposition rate of the fluid flow (Jha and Ajibade, 2009; Makinde and Beg, 2010; Hassan and Fenuga, 2011; Adesanya and Makinde, 2011; Hassan and Gbadeyan, 2015), this informed our study of the effects of internal heat generation within the flow system.

It is well known that the rate of heat transfer is temperature dependent, which increases the interaction of moving fluid and thus influence the internal energy of the flow regime. This interaction according to FrankKamenettski (1969), Makinde and Beg (2010) as well as Hassan and Gbadeyan (2014)bring about the condition of thermal runaway or ignition in the flow system to predict critical and unsafe situations.
The weighted residual method may be considered to be a unified version of a group of methods used to solve approximately boundary value, initial value and Eigen value problems. There are some simple and accurate approximation techniques for solving differential equations called the Weighted Residuals Methods (WRMs). Collocation, Galerkin and Least Square are examples of the WRMs. Stern and Rasmussen (1996) used collocation method for solving a third order linear differential equation. Vaferi et al. (2012) studied the feasibility of applying Orthogonal Collocation method to solve diffusivity equation in the radial transient flow system. Hendi and Albugami (2010) used Collocation and Galerkin methods for solving Fredholm-Volterra integral equation. Recently least square method was introduced by Aziz and Bouaziz (2011) and applied for it in predicting the performance of a longitudinal fin. They found that least squares method is simple compared with other analytical methods. Shaoqin and Huoyuan (2008) developed and analyzed least-squares approximations for the incompressible magneto-hydrodynamic equations. Recently, Hatami et al. (2014) studied the third grade non-Newtonian blood conveying gold nanoparticles in a porous and hollow vessel by two analytical methods called Least Square Method (LSM) and Galerkin Method (GM).

Hence, in the present study we will use the weighted residual method by collocation method to discuss an electrically conducting fluid with internal heat generation over a vertical plate on a reactive MHD fluid.

\section{Mathematical Formulation}

Consider a steady flow of an incompressible viscous fluid flow between two vertical Channelsof a reactive MHD flow withinternal heat generation. Let the $x$-axis be directed upward along the plate and the y-axis normal to the plate. The flow is electrically conducting in the presence of an applied magnetic field and is maintained due to a constant pressure gradient.

Let $u$ and $v$ be the velocity components along the $x$ - and $y$ - axes respectively. The continuity equation is given as

$\frac{\partial \bar{u}}{\partial \bar{x}}+\frac{\partial \bar{v}}{\partial \bar{y}}=0$ 
If the flow is in the $x$-direction, $\frac{\partial \bar{u}}{\partial \bar{x}}=0$, which implies $\frac{\partial \bar{v}}{\partial \bar{y}}=0$ Then the steady equations of motion (momentum and energy equation) for the system is presented by

$-\frac{\partial \bar{p}}{\partial \bar{x}}+v \frac{\partial^{2} \bar{u}}{\partial \bar{y}^{2}}-\frac{\sigma \mathrm{B}_{0}^{2} u}{\rho}+g \beta\left(T-T_{0}\right)=0(2)$

$\alpha \frac{\partial^{2} T}{\partial \bar{y}^{2}}+R a-\lambda\left(T-T_{0}\right)=0$

Where $\alpha$ is the thermal diffusivity, $v$ is the kinematic viscosity, $\beta$ is the volumetric expansion coefficient for heat transfer, $\rho$ is the density, $g$ is the acceleration due to gravity, $T$ is the temperature, $T_{0}$ is the reference or ambient temperature, $\lambda$ is the heat generation coefficient and $R a$ is the reaction term

With the following boundary conditions

$\bar{u}(0)=u_{0}, \quad \bar{u}(L)=0$.

$T(0)=T(L)=T_{0}$

$L$ is the width of the channel and $u_{0}$ is the velocity of the left plate.

\section{Dimensional Analysis}

By the scaling principle, we present the following dimensionless scales

$u=\frac{\bar{u}}{u_{0}}, y=\frac{\bar{y}}{L}, R a=Q C_{0} A \ell^{-\frac{E}{R T}}$ and $\theta=\frac{T-T_{0}}{\varepsilon T_{0}}(6)$

And reduced the dimensioned equations into dimensionless momentum and energy equations as

$P+\frac{\partial^{2} u}{\partial y^{2}}-N u+G r \theta=0$

$\frac{\partial^{2} \theta}{\partial y^{2}}-\lambda_{0} \theta+\lambda_{1} \ell^{\frac{\theta}{(\varepsilon \theta+1)}}=0$

With the following boundary conditions

$$
\begin{aligned}
& u(0)=1, u(1)=0 \\
& \theta(0)=\theta(1)=0
\end{aligned}
$$

Where, $N$ is the Hartman Number, Gris the Grash of Number, $P$ is the Pressure force, $\lambda_{0}$ is the Internal heat generation and $\lambda_{1}$ is the Arrhenius term.

\section{Weighted Residual Method (WRM) by Collocation method}

In this method, the weighting functions are taken from the family of Dirac $\delta$ functions in the domain. That is, $W_{i}(y)=\delta\left(y-y_{i}\right)$. The Dirac $\delta$ function has the property that

$$
\delta\left(y-y_{i}\right)= \begin{cases}1, & x=x_{i} \\ 0, & \text { otherwise }\end{cases}
$$

The idea in the WRM is to force the residual to zero in some average sense over the domain. That is

$\int_{Y} R(y) W_{i} d y=0, \quad i=1,2, \cdots, n$

where the number of weight functions $W_{i}$ is exactly equal the number of unknown constants $a_{i}$ in $\tilde{u}(y)$. The result is a set of $\mathrm{n}$ algebraic equations for the unknown constants $a_{i}$ (Salih, 2016).

the fixed points $y_{i} \in[a, b],(i=1,2, \cdot \cdots, n)$ are called collocation points. The number of collocation points selected must be equal to the number of unknown coefficients $a_{i}{ }^{\prime} s$ in the definition of approximating function, $u(y)$.

Hence, the integration of the weighted residual statement results in the forcing of the residual to zero at specific points in the domain. That is, integration of (12) with $W_{i}(y)=\delta\left(y-y_{i}\right)$ results in $R\left(y_{i}\right)=0$

Thus, by the collocation method, we will use a polynomial function as a basis. That is, let the approximating function $u(y)$ which can be chosen arbitrary be

$$
\left.\begin{array}{l}
u(y)=a_{0}+a_{1} y+a_{2} y^{2}+a_{3} y^{3}+a_{4} y^{4} \\
\theta(y)=b_{0}+b_{1} y+b_{2} y^{2}+b_{3} y^{3}+b_{4} y^{4}
\end{array}\right\}
$$

\section{Solution to the dimensionless momentum and energy equations using the collocation method}

We will chose the trial functions in such a way that the assumed function $\tilde{u}(y)$ satisfies the global boundary conditions for (13) Thus, 


$$
\begin{aligned}
u(y)= & 1-y^{4}+a_{1} y\left(1-y^{3}\right) \\
& +a_{2} y^{2}\left(1-y^{2}\right)+a_{3} y^{3}(1-y)
\end{aligned}
$$

and

$$
\theta(y)=b_{1} y\left(1-y^{3}\right)+b_{2} y^{2}\left(1-y^{2}\right)+b_{3} y^{3}(1-y)(15)
$$

Hence,

$$
\begin{aligned}
& R_{1}(y)=P+\left(\begin{array}{l}
-12 y^{2}-12 a_{1} y^{2}+2 a_{2}\left(1-12 y^{2}\right) \\
+6 a_{3} y(1-2 y)
\end{array}\right) \\
& -N\left(\begin{array}{l}
1-y^{4}+a_{1} y\left(1-y^{3}\right)+a_{2} y^{2}\left(1-y^{2}\right) \\
+a_{3} y^{3}(1-y)
\end{array}\right) \\
& +G r\left(\begin{array}{l}
b_{1} y\left(1-y^{3}\right)+b_{2} y^{2}\left(1-y^{2}\right) \\
+b_{3} y^{3}(1-y)
\end{array}\right) \\
& R_{2}(y)=\left(\begin{array}{l}
-12 b_{1} y^{2}+2 b_{2}\left(1-6 y^{2}\right) \\
+6 b_{3} y(1-2 y)
\end{array}\right) \\
& -\lambda_{0}\left(\begin{array}{l}
b_{1} y\left(1-y^{3}\right)+b_{2} y^{2}\left(1-y^{2}\right) \\
+b_{3} y^{3}(1-y)
\end{array}\right) \\
& \left(b_{1} y\left(1-y^{3}\right)+b_{2} y^{2}\left(1-y^{2}\right)+b_{3} y^{3}(1-y)\right) \\
& +\lambda_{1} \ell^{\left(\varepsilon\left(b_{1} y\left(1-y^{3}\right)+b_{2} y^{2}\left(1-y^{2}\right)+b_{3} y^{3}(1-y)\right)+1\right)}
\end{aligned}
$$

Evaluating equations (16) and (17) at the following collocation points $(y=0.25, y=0.5, y=0.75)$ gives the following relations.

$$
\begin{aligned}
& R_{1}(0.25)=P-0.7500 a_{1}+1.2500 a_{2}+0.7500 a_{3}-0.7500 \\
& -N\left(\begin{array}{l}
0.99609375+0.24609375 a_{1} \\
+0.05859375 a_{2}+0.01171875 a_{3}
\end{array}\right) \\
& +G r\left(\begin{array}{l}
0.2460937 \boldsymbol{b}_{1}+0.0585937 \boldsymbol{b}_{2} \\
+0.0117187 \boldsymbol{b}_{3}
\end{array}\right)=0 \\
& R_{2}(0.25)=-0.7500 b_{1}+1.2500 b_{2}+0.7500 b_{3} \\
& -\lambda_{0}\left(\begin{array}{l}
0.2460937 \boldsymbol{b}_{1}+0.0585937 \boldsymbol{b}_{2} \\
+0.0117187 \boldsymbol{\not}_{3}
\end{array}\right) \\
& +\lambda_{1} e^{\frac{\left(0.24609375 b_{1}+0.05859375 b_{2}+0.01171875 b_{3}\right)}{\varepsilon\left(0.24609375 b_{1}+0.05859375 b_{2}+0.01171875 b_{3}\right)+1}}=0
\end{aligned}
$$




$$
\begin{aligned}
& R_{1}(0.5)=P-3.00 a_{1}-1.00 a_{2}-3.00 \\
& -N\left(\begin{array}{l}
0.9375+0.4375 a_{1}+0.1875 a_{2} \\
+0.0625 a_{3}
\end{array}\right) \\
& +G r\left(\begin{array}{l}
0.4375 b_{1}+0.1875 b_{2} \\
+0.0625 b_{3}
\end{array}\right)=0 \\
& R_{2}(0.5)=-3.00 b_{1}-1.00 b_{2} \\
& -\lambda_{0}\left(0.4375 b_{1}+0.1875 b_{2}+0.0625 b_{3}\right) \\
& +\lambda_{1} e^{\frac{\left(0.4375 b_{1}+0.1875 b_{2}+0.0625 b_{3}\right)}{\varepsilon\left(0.4375 b_{1}+0.1875 b_{2}+0.0625 b_{3}\right)+1}}=0 \\
& R_{1}(0.75)=P-6.7500 a_{1}-4.7500 a_{2} \\
& -2.2500 a_{3}-6.7500 \\
& -N\left(\begin{array}{l}
0.68359375+0.43359375 a_{1} \\
+0.24609375 a_{2}+0.10546875 a_{3}
\end{array}\right) \\
& +G r\left(\begin{array}{l}
0.4335937 \boldsymbol{B}_{1}+0.2460937 \boldsymbol{B}_{2} \\
+0.1054687 \boldsymbol{b}_{3}
\end{array}\right)=0 \\
& R_{2}(0.75)=-6.7500 b_{1}-4.7500 b_{2}-2.2500 b_{3} \\
& -\lambda_{0}\left(\begin{array}{l}
0.4335937 \boldsymbol{b}_{1}+0.2460937 \boldsymbol{b}_{2} \\
+0.1054687 \boldsymbol{b}_{3}
\end{array}\right) \\
& +\lambda_{1} e^{\frac{\left(0.43359375 b_{1}+0.24609375 b_{2}+0.10546875 b_{3}\right)}{\varepsilon\left(0.43359375 b_{1}+0.24609375 b_{2}+0.10546875 b_{3}\right)+1}}=0
\end{aligned}
$$

The $a_{i}{ }^{\prime} s$ and $b_{i}{ }^{\prime} s$ are obtained from solving (18), (19) and (20) as a system of linear equations with suitable values of the dimensionless constants and with the help of maple 17 software we summarize the solution in the table together with the numerical solution of the momentum and energy equations as follows.

Table1: Coefficients of the Basis Functions with Increasing Internal Heat Generation $\lambda_{0}$ and $(-P=1, N=0.2)$, $\left(G r=0.1, \varepsilon=0.5\right.$ and $\left.\lambda_{1}=1.2\right)$

\begin{tabular}{|l|l|l|l|l|l|l|}
\hline$\lambda_{0}$ & $a_{1}$ & $a_{2}$ & $a_{3}$ & $b_{1}$ & $b_{2}$ & $b_{3}$ \\
\hline 1.2 & -1.553547322 & 0.5994305394 & -0.05864014171 & 0.5051908141 & -0.5901416525 & 0.1699016768 \\
\hline 1.4 & -1.553609463 & 0.5994166552 & -0.05848808951 & 0.4985351037 & -0.5895618927 & 0.1820535781 \\
\hline 1.6 & -1.553669635 & 0.5994033487 & -0.05834113393 & 0.4920813209 & -0.5889325390 & 0.1937024362 \\
\hline
\end{tabular}

Table 2: Coefficients of the Basis Functions with Increasing Reactive Rate $\lambda_{1}$ and $(-P=1, N=0.2, G r=0.1)$, $\left(\varepsilon=0.5\right.$ and $\left.\lambda_{0}=1.2\right)$

\begin{tabular}{|l|l|l|l|l|l|l|}
\hline$\lambda_{1}$ & $a_{1}$ & $a_{2}$ & $a_{3}$ & $b_{1}$ & $b_{2}$ & $b_{3}$ \\
\hline 1.2 & -1.553547322 & 0.5994305394 & -0.05864014171 & 0.5051908141 & -0.5901416525 & 0.1699016768 \\
\hline 1.4 & -1.552936843 & 0.5993847083 & -0.05976943689 & 0.5827246268 & -0.6861007858 & 0.2067523180 \\
\hline 1.6 & -1.552340174 & 0.5993371739 & -0.06086770593 & 0.6586871810 & -0.7812463833 & 0.2451184046 \\
\hline
\end{tabular}


Table 3: Coefficients of the Basis Functions with Varying Hartman Number $N$ and $\left(-P=1, \lambda_{1}=1.2, G r=0.1\right)$, $\left(\varepsilon=0.5\right.$ and $\left.\lambda_{0}=1.2\right)$

\begin{tabular}{|l|l|l|l|l|l|l|}
\hline$N$ & $a_{1}$ & $a_{2}$ & $a_{3}$ & $b_{1}$ & $b_{2}$ & $b_{3}$ \\
\hline 1 & -1.552695423 & 0.5996078885 & -0.06069863735 & 0.5972991736 & -0.6045225430 & 0.01444673890 \\
\hline 2 & -2.012818193 & 1.464122307 & -0.5888827392 & 0.5972991736 & -0.6045225430 & 0.01444673890 \\
\hline 3 & -2.231801428 & 1.919487300 & -0.9182288879 & 0.5972991736 & -0.6045225430 & 0.01444673890 \\
\hline
\end{tabular}

Table 4: Coefficients of the Basis Functions with Varying Pressure $P$ and $\left(N=0.2, \lambda_{1}=1.2, G r=0.1\right)$, $\left(\varepsilon=0.5\right.$ and $\left.\lambda_{0}=1.2\right)$

\begin{tabular}{|l|l|l|l|l|l|l|}
\hline$P$ & $a_{1}$ & $a_{2}$ & $a_{3}$ & $b_{1}$ & $b_{2}$ & $b_{3}$ \\
\hline 1 & -1.769224197 & 0.9910133792 & -0.2819622032 & 0.5999624688 & -0.6002005634 & 0.0004761892805 \\
\hline 2.5 & -2.462201562 & 1.739707517 & -0.3933957494 & 0.5999624688 & -0.6002005634 & 0.0004761892805 \\
\hline 5 & -3.617163837 & 2.987531081 & -0.5791183263 & 0.5999624688 & -0.6002005634 & 0.0004761892805 \\
\hline
\end{tabular}

Table 5: Coefficients of the Basis Functions with Varying Grashorf Number $G r$ and $\left(N=0.2, \lambda_{1}=1.2, P=1\right)$, $\left(\varepsilon=0.5\right.$ and $\left.\lambda_{0}=1.2\right)$

\begin{tabular}{|l|l|l|l|l|l|l|}
\hline$G r$ & $a_{1}$ & $a_{2}$ & $a_{3}$ & $b_{1}$ & $b_{2}$ & $b_{3}$ \\
\hline 0.1 & -1.769224197 & 0.9910133792 & -0.2819622032 & 0.5999624688 & -0.6002005634 & 0.0004761892805 \\
\hline 0.5 & -1.750979201 & 09905933739 & -0.3176121851 & 0.5999624688 & -0.6002005634 & 0.0004761892805 \\
\hline 1 & -1.728172955 & 0.9900683672 & -0.3621746624 & 0.5999624688 & -0.6002005634 & 0.0004761892805 \\
\hline
\end{tabular}

Thus, by the collocation method, solution of the momentum and energy equations are given by

$$
\begin{gathered}
u_{\lambda_{0}=1.2}(y)=\left(1-y^{4}\right)-1.55354732 y\left(1-y^{3}\right) \\
+0.599430534 y^{2}\left(1-y^{2}\right) \\
-0.0586401171 y^{3}(1-y) \\
u_{\lambda_{0}=1.4}(y)=\left(1-y^{4}\right)-1.55360946 y\left(1-y^{3}\right) \\
+0.599416652 y^{2}\left(1-y^{2}\right) \\
-0.0584880851 y^{3}(1-y) \\
u_{\lambda_{0}=1.6}(y)=\left(1-y^{4}\right)-1.553669635 y\left(1-y^{3}\right) \\
+0.5994033487 y^{2}\left(1-y^{2}\right) \\
-0.05834113393 y^{3}(1-y)
\end{gathered}
$$

and

$$
\begin{array}{r}
\theta_{\lambda_{0}=1.2}(y)=0.5051908141 y\left(1-y^{3}\right) \\
-0.590141625 y^{2}\left(1-y^{2}\right) \\
+0.1699016768 y^{3}(1-y)
\end{array}
$$


206| The Effect of Internal Heat Generation of a Reactive Hydromagnetic (MHD)...

$$
\begin{aligned}
\theta_{\lambda_{0}=1.4}(y) & =0.4985351037 y\left(1-y^{3}\right) \\
- & 0.5895618927 y^{2}\left(1-y^{2}\right) \\
+ & 0.1820535781 y^{3}(1-y) \\
\theta_{\lambda_{0}=1.6}(y) & =0.4920813209 y\left(1-y^{3}\right) \\
- & 0.5889325390 y^{2}\left(1-y^{2}\right) \\
+ & 0.1937024362 y^{3}(1-y)
\end{aligned}
$$

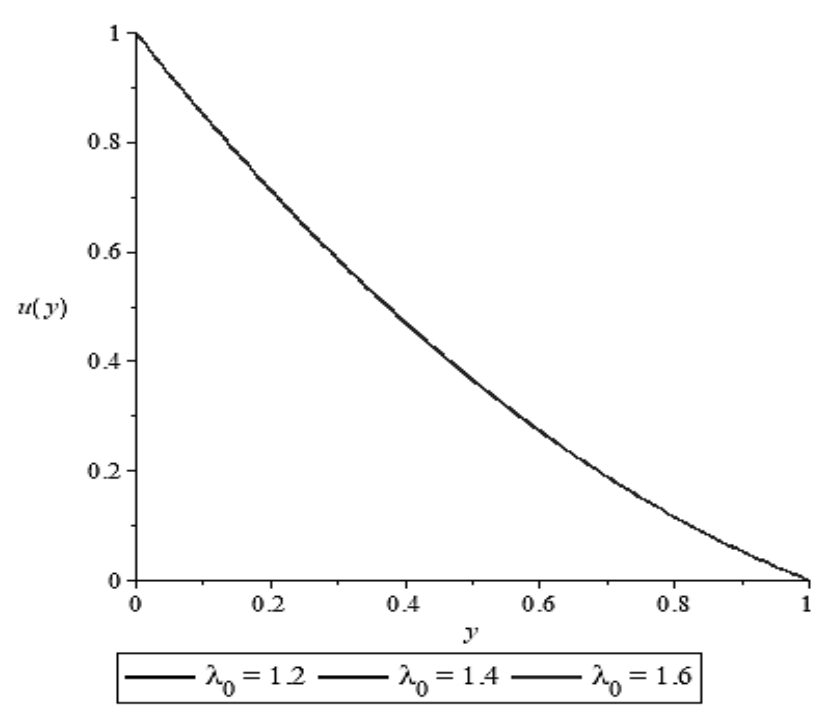

Figure 1: Effect of the Increasing Internal HeatGeneration $\lambda_{0}$ on the Velocity Profile.

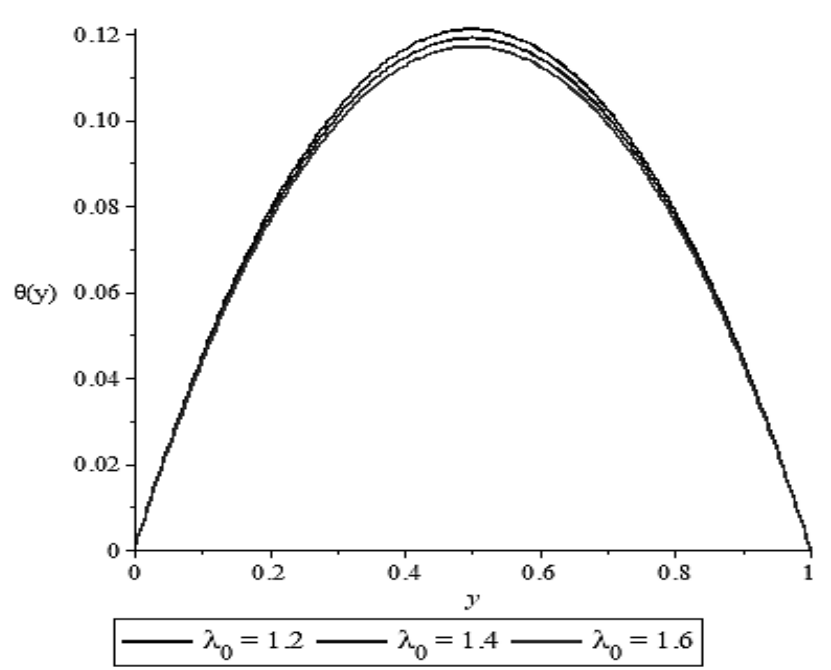

Figure 2: Effect of the Increasing Internal Heat Generation $\lambda_{0}$ on the Temperature profile.

$$
\begin{aligned}
u_{\lambda_{1}=1.2}(y) & =\left(1-y^{4}\right)-1.55354732 y\left(1-y^{3}\right) \\
& +0.5994305394 y^{2}\left(1-y^{2}\right) \\
& -0.05864014171 y^{3}(1-y)
\end{aligned}
$$




$$
\begin{aligned}
& u_{\lambda_{1}=1.4}(y)=\left(1-y^{4}\right)-1.55293684 B y\left(1-y^{3}\right) \\
& +0.5993847883 y^{2}\left(1-y^{2}\right) \\
& -0.05976943689 y^{3}(1-y) \\
& u_{\lambda_{1}=1.6}(y)=\left(1-y^{4}\right)-1.55234017 y y\left(1-y^{3}\right) \\
& +0.5993371 B 9 y^{2}\left(1-y^{2}\right) \\
& -0.06086770993 y^{3}(1-y) \\
& \theta_{\lambda_{1}=1.2}(y)=0.5051908141 y\left(1-y^{3}\right) \\
& -0.590141655 y^{2}\left(1-y^{2}\right) \\
& +0.1699016768 y^{3}(1-y) \\
& \theta_{\lambda_{1}=1.4}(y)=0.5827246268 y\left(1-y^{3}\right) \\
& -0.6861007858 y^{2}\left(1-y^{2}\right) \\
& +0.2067523180 y^{3}(1-y) \\
& \theta_{\lambda_{1}=1.6}(y)=0.6586871810 y\left(1-y^{3}\right) \\
& -0.781246383 y^{2}\left(1-y^{2}\right) \\
& +0.2451184046 y^{3}(1-y)
\end{aligned}
$$

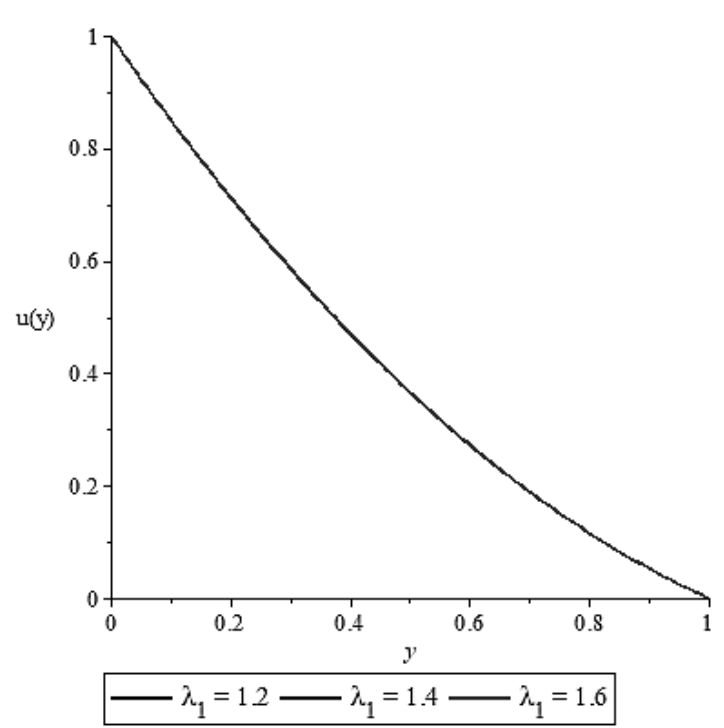

Figure 3: Effect of the Reactive Rate $\lambda_{1}$ on the velocity Profile. 
208| The Effect of Internal Heat Generation of a Reactive Hydromagnetic (MHD)...

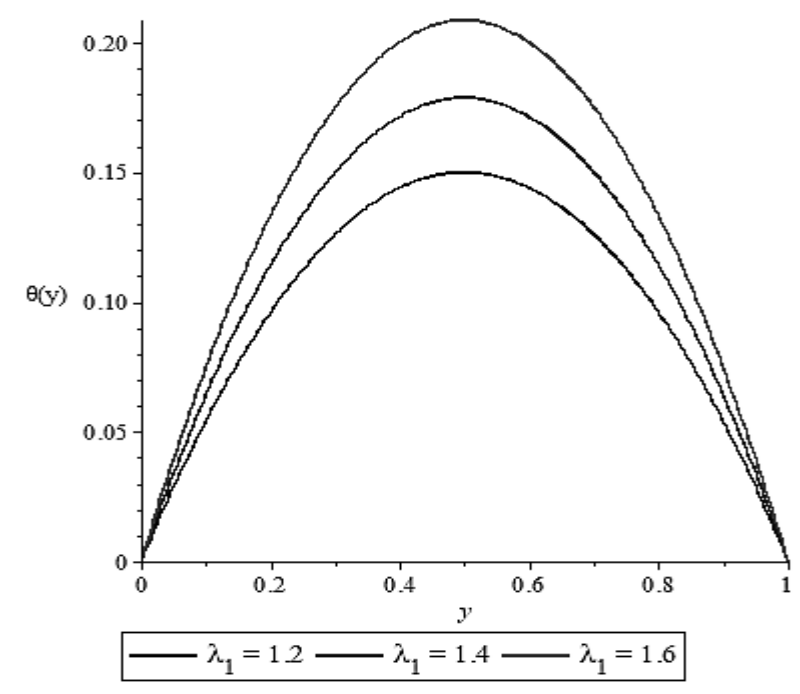

Figure 4: Effect of the Reactive Rate $\lambda_{1}$ on theTemperatureProfile

$$
\begin{aligned}
u_{N=1}(y) & =\left(1-y^{4}\right)-1.552695423 y\left(1-y^{3}\right) \\
& +0.599607885 y^{2}\left(1-y^{2}\right) \\
& -0.06069863735 y^{3}(1-y) \\
u_{N=2}(y) & =\left(1-y^{4}\right)-2.01281818 y\left(1-y^{3}\right) \\
& +1.464122307 y^{2}\left(1-y^{2}\right) \\
& -0.5888827322 y^{3}(1-y)
\end{aligned}
$$

$$
\begin{aligned}
u_{N=3}(y) & =\left(1-y^{4}\right)-2.231801428 y\left(1-y^{3}\right) \\
& +1.91948730 y^{2}\left(1-y^{2}\right) \\
& -0.9182288879 y^{3}(1-y)
\end{aligned}
$$

and

$$
\begin{aligned}
\theta_{N=1}(y) & =0.5972991 \mathcal{B} 6 y\left(1-y^{3}\right) \\
& -0.6045225430 y^{2}\left(1-y^{2}\right) \\
& +0.01444673890 y^{3}(1-y)
\end{aligned}
$$

$$
\begin{aligned}
\theta_{N=2}(y) & =0.5972991 B 6 y\left(1-y^{3}\right) \\
& -0.604522540 y^{2}\left(1-y^{2}\right) \\
& +0.01444673890 y^{3}(1-y)
\end{aligned}
$$

$$
\begin{aligned}
\theta_{N=3}(y) & =0.5972991 B 6 y\left(1-y^{3}\right) \\
& -0.604522540 y^{2}\left(1-y^{2}\right) \\
& +0.01444673890 y^{3}(1-y)
\end{aligned}
$$




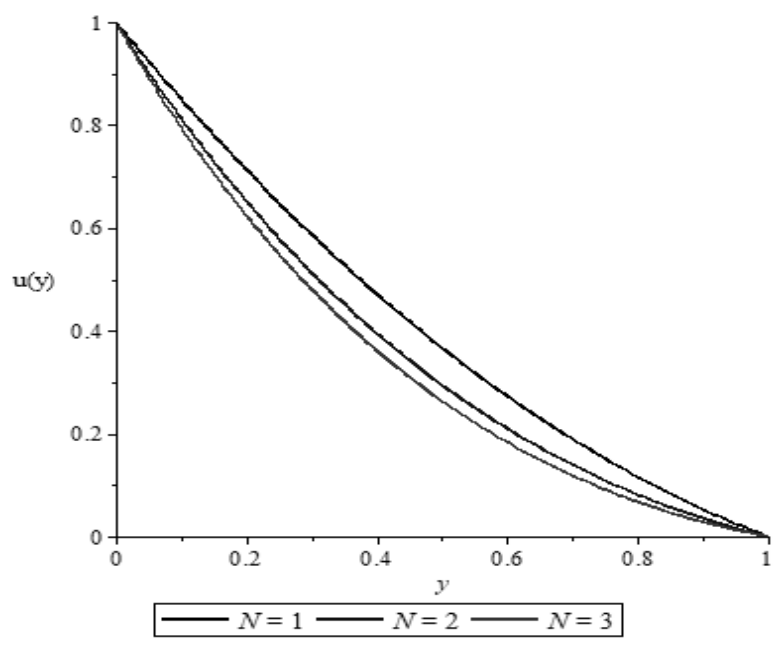

Figure 5: Effect of the Hartman Number $N$ onthe Velocity Profile

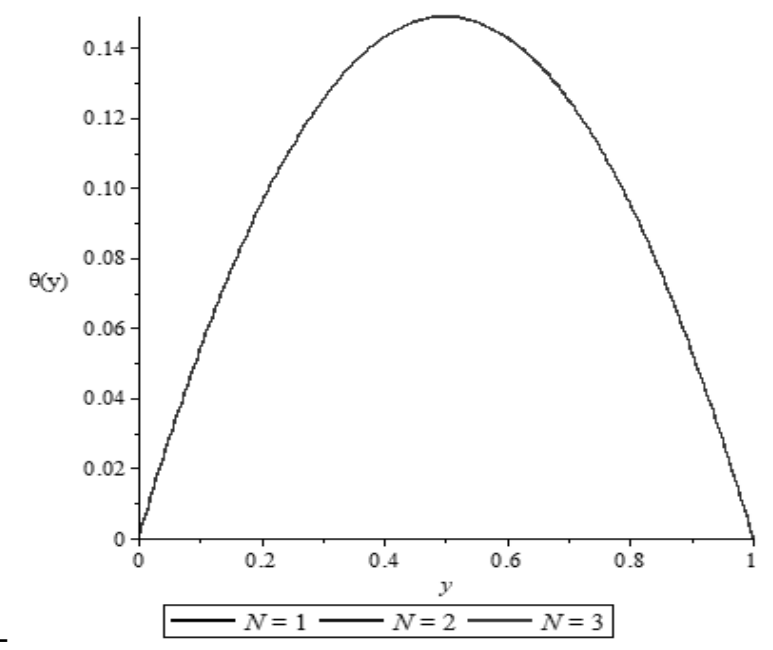

Figure 6: Effect of the Hartman Number $N$ on the Temperature Profile

$$
\begin{aligned}
u_{P=1}(y) & =\left(1-y^{4}\right)-1.76922419 y\left(1-y^{3}\right) \\
& +0.991013372 y^{2}\left(1-y^{2}\right) \\
& -0.281962202 y^{3}(1-y) \\
u_{P=2.5}(y) & =\left(1-y^{4}\right)-2.4622015 Q y\left(1-y^{3}\right) \\
& +1.73970757 y^{2}\left(1-y^{2}\right) \\
& -0.393395744 y^{3}(1-y) \\
u_{P=5}(y) & =\left(1-y^{4}\right)-3.61716383 y\left(1-y^{3}\right) \\
& +2.987531081 y^{2}\left(1-y^{2}\right) \\
& -0.579118363 y^{3}(1-y)
\end{aligned}
$$

and

$$
\begin{aligned}
\theta_{P=1}(y) & =0.599962488 y\left(1-y^{3}\right) \\
& -0.600200564 y^{2}\left(1-y^{2}\right) \\
& +0.000476182805 y^{3}(1-y)
\end{aligned}
$$




$$
\begin{aligned}
\theta_{P=2.5}(y) & =0.599962488 y\left(1-y^{3}\right) \\
- & 0.600200564 y^{2}\left(1-y^{2}\right) \\
+ & 0.000476182805 y^{3}(1-y) \\
\theta_{P=5}(y) & =0.599962488 y\left(1-y^{3}\right) \\
& -0.600200564 y^{2}\left(1-y^{2}\right) \\
& +0.000476182805 y^{3}(1-y)
\end{aligned}
$$

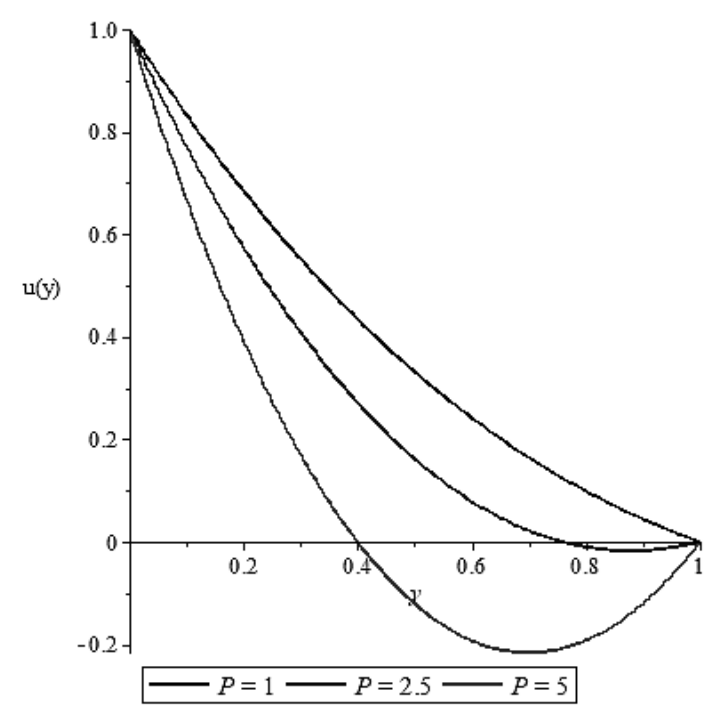

Figure 7: Effect of the Pressure $P$ on the Velocity Profile

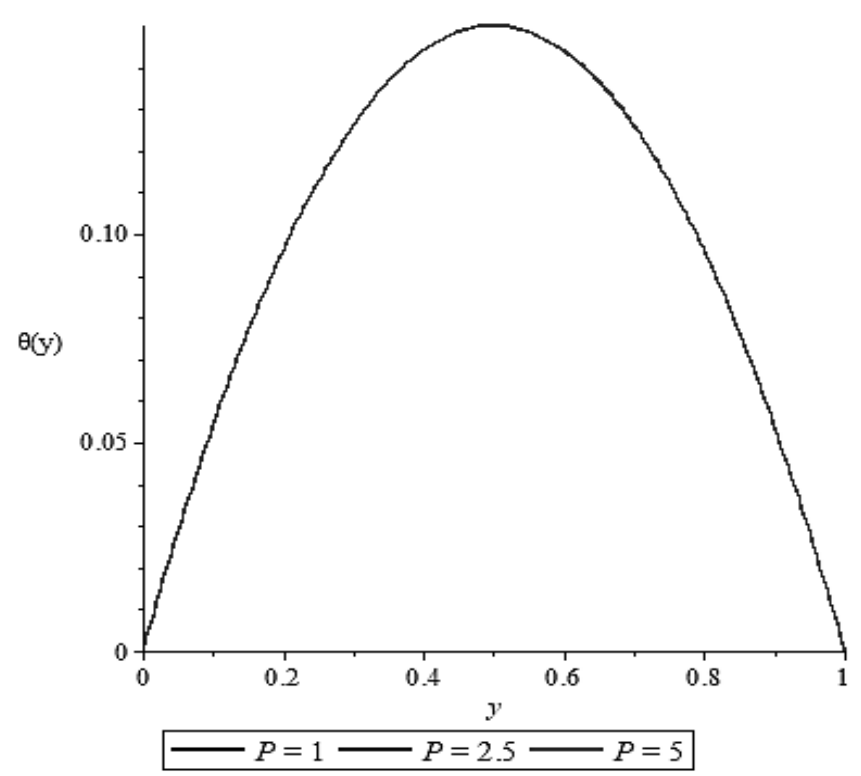

Figure 8: Effect of the Pressure $P$ on the TemperatureProfile

$$
\begin{gathered}
u_{G r=0.1}(y)=\left(1-y^{4}\right)-1.76922419 y\left(1-y^{3}\right) \\
+0.9910133792 y^{2}\left(1-y^{2}\right) \\
-0.2819622\left(B 2 y^{3}(1-y)\right.
\end{gathered}
$$




$$
\begin{aligned}
u_{G r=0.5}(y) & =\left(1-y^{4}\right)-1.75097920 y\left(1-y^{3}\right) \\
& +0990593373 y^{2}\left(1-y^{2}\right) \\
& -0.3176121851 y^{3}(1-y) \\
u_{G r=1}(y) & =\left(1-y^{4}\right)-1.72817295 y\left(1-y^{3}\right) \\
+ & 0.990068362 y^{2}\left(1-y^{2}\right) \\
& -0.362174624 y^{3}(1-y)
\end{aligned}
$$

and

$$
\begin{aligned}
\theta_{G r=0.1}(y) & =0.599962488 y\left(1-y^{3}\right) \\
& -0.600200564 y^{2}\left(1-y^{2}\right) \\
& +0.000476182805 y^{3}(1-y)
\end{aligned}
$$

$$
\begin{aligned}
\theta_{G r=0.5}(y) & =0.599962488 y\left(1-y^{3}\right) \\
& -0.600200564 y^{2}\left(1-y^{2}\right) \\
+ & 0.000476182805 y^{3}(1-y)
\end{aligned}
$$

$$
\begin{aligned}
\theta_{G r=1}(y) & =0.599962488 y\left(1-y^{3}\right) \\
& -0.600200564 y^{2}\left(1-y^{2}\right) \\
& +0.000476192805 y^{3}(1-y)
\end{aligned}
$$

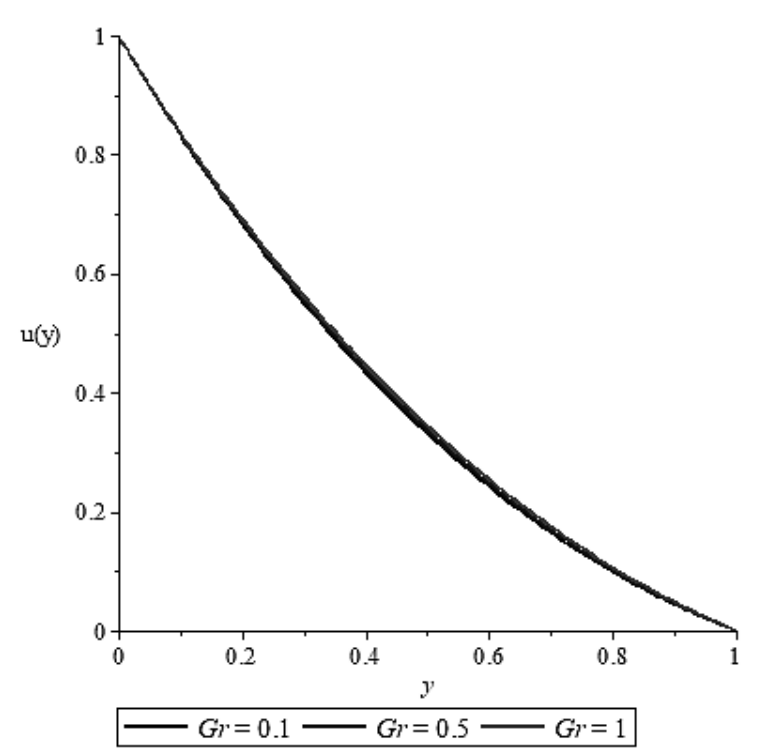

Figure 9: Effect of the Grashorf Number $G r$ onthe VelocityProfile 


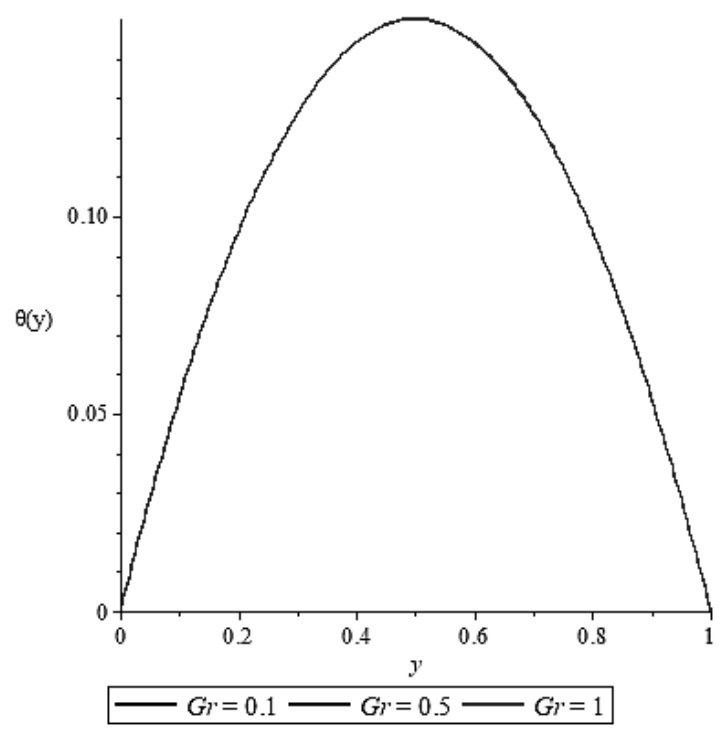

Figure 10: Effect of the Grashorf Number $G r$ on the Temperature Profile

\section{Discussion}

We will discuss the effects of the internal heat generation together with other important flow parameters on the hydromagnetic fluid flow between two vertical plates with an isothermal wall temperature in this session. These are presented in tables and graphs using Maple 17 Mathematical software.

Figure 1 and 2 shows the effect of the increasing internal heat generation $\lambda_{0}$ on the velocity and temperature profile respectively. Increase in the internal heat generation parameter $\lambda_{0}$ reduces the temperature of the fluid and has no significant effect on the velocity profile while increase in the Reactive Rate $\lambda_{1}$ increases the temperature and shows no significant change in the velocity profile as can be seen by Figure 3 and 4.Increase in the Stuart Number $N$ reduces the velocity of the flow. The Stuart Number $N$ which is also known as the magnetic interaction parameter which is the ratio of electromagnetic to inertial forces on flow have no significant effect on the temperature profile as can be seen from figures 5 and 6 respectively. Figures 7 and 8 shows that the pressure forces $P$ which drives the flow also reduces the velocity and even goes beyond the boundary line at higher pressure forces while the temperature has no significant change with increase in the pressure forces. The Grashof Number $G r$ which is a dimensionless number in fluid dynamics and heat transfer approximates the ratio of the buoyancy to the viscous force acting on a fluid, increases with increase in the velocity profile. It however does not affect the temperature as seen by figures 9 and 10.

\section{Conclusion}

The effect of internal heat generation on a steady hydromagnetic fluid flow between two Channels is investigated. Analytical solutions of the equations governing the fluid flow are obtained using the method of weighted residual by collocation method. In view of the above results, the following are the major contributions in this study:

i. Theeffect of the increasing Stuart Number $N$ and the pressure $P$ reduces the velocity while the increasing Grashorf Number $G r$ increases the fluid velocity and the temperature remains indifferent.

ii. Due to collision of the fluid particles, internal heat $\lambda_{0}$ is being generated which eventually leads to a decrease in the fluid temperature.

iii. The temperature increases as the reactive rate $\lambda_{1}$ which is the modification of the Arrhenius Number increases while the velocity remains indifferent.

Finally, we showed that the internal heat generation, magnetic field intensity and the reactive rate have significant effects on the velocity profiles and temperature distributions.

\section{References}

Makinde, O. D. and Beg, O. A. (2010) On Inherent Irreversibility in a Reactive Hydromagnetic Channel 
Flow. Journal of Thermal Science 19 (1):72-79.

Hassan, A. R. and Gbadeyan, J. A. (2015) Entropy Generation Analysis of a Reactive Hydromagnetic Fluid Flow Through a Channel. UnivPolitech BucharSci Bull SerA 77(2):285-296

Frank-Kamenettski, D. A. (1969) Diffusion and Heat Transfer in Chemical Kinetics. Plenum Press, New York

Hassan, A. R. and Gbadeyan, J. A. (2014) Thermal Stability Analysis of a Reactive Hydromagnetic Fluid Flow Through a Channel. American Journal of Applied Mathematics 2(1):14-20

Hassan, A. R and Gbadeyan, J. A. (2015). A Reactive Hydromagnetic Internal Heat Generating Fluid Flow Through a Channel. International Journal of Heat and Technology. 33(3):43-50.

Jha, B. K. and Ajibade, A. O. (2009). Free Convective Flow of Heat Generating/Absorbing Fluid between Vertical Porous Plates with Periodic Heat Input. Journal of International Communal Heat and Mass Transfer. 36:624-631.

Aziz, A. and Bouaziz, M.N. (2011).A Least Squares Method for a Longitudinal Fin with Temperature Dependent Internal Heat Generation and Thermal Conductivity. Energy Conversion and Management.52: 2876-82.

Hassan, A. R. and Fenuga, O. J. (2011). Flow of a Maxwell Fluid Through a Porous
Medium Induced by a Constantly Accelerating Plate. Journal of Nigerian Association of Mathematical Physics 19:249-254

Stern, R.H. and Rasmussen, H. (1996). Left Ventricular Ejection: Model Solution by Collocation, an Approximate Analytical Method. Computers in Biology and Medicine.26: 255-61.

Vaferi, B., Salim,i V., Dehghan B. D., Jahanmiri, A. and Khedri, S. (2012). Prediction of Transient Pressure Response in the Petroleum Reservoirs using Orthogonal Collocation. Journal of Petroleum Science and Engineering. 98-99:156-63.

Hendi, F.A. and Albugami, A.M. (2010). Numerical Solution for FredholmVolterra Integral Equation of the second kind by using Collocation and Galerkin methods. Journal of King Saud University -Science.22:37-40.

Hatami, M., Hatami, J. and Ganji, D. D. (2014). Computer Simulation of MHD Blood Conveying Gold Nanoparticles as a Third Grade Non-Newtonian Nanofluid in a Hollowporous Vessels. Computer Method and Program in Biomedicine.113: 632-641.

Shaoqin, G. and Huoyuan, D. (2008).Negative Norm Least-squares Methods for the Incompressible Magneto-hydrodynamic Equations. Acta Mathematics Scientia.28 $675-84$. 